\title{
Finding the Smallest Circle Containing The Iris in The Denoised Wavelet Domain
}

\author{
Pablo Rivas-Perea*, Ryan Henning*, Bryan Shaw ${ }^{\dagger}$ and Greg Hamerly* \\ * Department of Computer Science \\ ${ }^{\dagger}$ Department of Chemistry \\ Baylor University \\ Waco, TX, USA \\ Contact Info: http://leuko.net/
}

\begin{abstract}
Retinoblastoma is a pediatric ocular cancer typically indicated by leukocoria (white-eye pupillary reflex). Early detection of leukocoria can improve health outcomes when it indicates disease, and it can be easily seen in recreational photographs. As part of a system for automatic leukocoria detection, we propose an image processing algorithm for detecting the exact location and radius of the smallest circle containing the iris in an eye image. Our algorithms use both median filters and two-dimensional stationary wavelet transforms and achieve low error rates.
\end{abstract}

Keywords-iris; median filter; wavelets; leukocoria; retinoblastoma

\section{INTRODUCTION}

Leukocoria is a white pupillary reflex defined as abnormal reflection of light in the retina of the eye [1]. It is similar, in appearance, to the reflection observed in creatures that have a tapetum lucidum, e.g., cats, dogs, and raccoons. The human eye does not have a tapetum lucidum, and if leukocoria is observed in the human eye this is typically interpreted as a negative event. Leukocoria often indicates medical conditions that include, but are not limited to, retinoblastoma, Coats disease, congenital cataract, corneal scarring, among several others [2]. Retinoblastoma is a pediatric ocular cancer that can be life-threatening if not treated in a timely manner.

Leukocoria is detectable using a conventional camera with a flash. In a photograph, the healthy human eye should appear to have a strong red reflection or no reflection at all. However, the leukocoric eye appears with a white or orange colored reflection [3]. An ophthalmologist typically uses medical instrumentation to confirm the existence of leukocoria. Despite of the simplicity of leukocoria detection, there are no automated detection systems that operate on conventional digital photographs. Such a system needs to locate faces, eyes, and even pupils within an arbitrary image.

This paper proposes an image processing and analysis algorithm to identify the exact center and radius of the smallest circle containing the iris within the image of an eye for further analysis. The image processing involves denoising the input image to produce an image that is better suited for edge detection over relatively large regions with respect to the input size. The first image processing strategy is a median filter that is proportional to the size of the input image. The second strategy is a two-dimensional discrete stationary wavelet (2D SWT) approach for denoising of the input image. The latter strategy has been successfully used in denoising images with high signal to noise ratio [4]. The proposed algorithm proceeds to find the smallest circle that contains the iris in the enhanced image using the Hough transform (HT) for circles. This transformation makes use of an edge image and creates a set of matrices containing the votes for particular circle sizes centered at specific image locations [5]. The HT has been widely used in the problem of circle detection [6], but not specifically in the application presented in this paper.

\section{Datasets And Image Pre-Processing}

This research uses ten different groups of images totaling 704 color images. Six groups contain 70 images each, while the remaining four groups have 71 images each. The groups were created in this manner to ease the estimation of a $K$ fold-based cross-validation error. All images are squared, varying their size from $19 \times 19$ to $138 \times 138$ pixels. Most images are not centered with respect to the iris, but some may be unintentionally close to the center. Orientation of the images also varies and the eye may not contain a perfect circle for the cases when the picture is captured in angle with respect to the camera sensor. The eye is usually not entirely open causing incomplete iris circles. The color of the eye and the texture and color of the skin also vary. All images were extracted from recreational photographs, thus, many lack proper illumination, resolution, and quality. Consider Figure 1 which shows representative examples of the images that conform the datasets. Such images are labeled and manually tagged with the exact center $\left(c_{1}, c_{2}\right)$ and radius $r$ of the smallest circle that contains the iris of the image, where $c_{1} \in\{0,1, \ldots, M-1\}$ and $c_{2} \in\{0,1, \ldots, N-1\}$ for an $M \times N$ image. This information is used to compute the error in the detection methods.

Evidently, recreational photography produces digital images that are likely to have no control over the illumination of the scene. Usually, the camera flash produces uneven illumination and color artifacts inside the eye. But more importantly, the different skin colors may introduce un- 


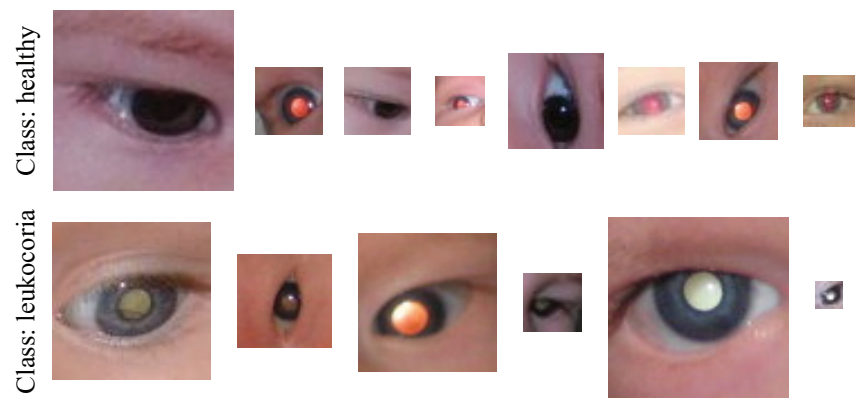

Figure 1. Sample images of the image datasets used in this research. Images vary in size, orientation, skin color, iris color, and angle of the eye with respect to the camera.

wanted information into the classification system. In order to avoid costly skin-detection algorithms that could be used to remove (ignore) skin regions, we simply equalize the histogram of the cropped eye image at each channel. This equalization of channel histogram alleviates the necessity of evening the image and reduces the effects of skin color in the eye image. Nonetheless, the equalization of the histograms needs to be performed after a process of denoising. In this manner, there will be more benefit from the equalization process. The two denoising methodologies used are explained in the next section.

\section{IMAGE ANALYSIS}

Part of the issues in amateur photography is the increase of noise in proportion to the lack of illumination. Furthermore, since we are interested only in the center of the eye, i.e., in detecting the circular region containing the iris, we can perform noise-reduction up to the point in which the region containing the iris is still clearly delimited. In this research we use two approaches that reduce the noise in the image and preserve the limits of the region of interest. These are briefly explained next.

\section{A. Median filtering}

A 2D median filter is widely used in the image processing community because it preserves edges and removes speckle noise fairly well. An $M \times N$ input image $\mathbf{I}\left(n_{1}, n_{2}\right)$, where $n_{1} \in\{0,1, \ldots, M-1\}$ and $n_{2} \in\{0,1, \ldots, N-1\}$, can be filtered considering an $p \times q$ neighborhood, where the neighborhood is usually $1 \leq p \ll M$ and $1 \leq q \ll N$. Every pixel considered as being filtered observes its neighbors and replaces itself with the median value of its neighborhood.

In this research, we propose a square filter whose size is dependent on the image size in an quadratic relationship. The proposed $p \times p$ filter can be used obtaining its size as follows:

$$
p=\lceil\sqrt{\max (M, N)}\rceil .
$$

After the estimation of the filter size we proceed to apply the $p \times p$ median filter to the three (i.e., RGB) channels

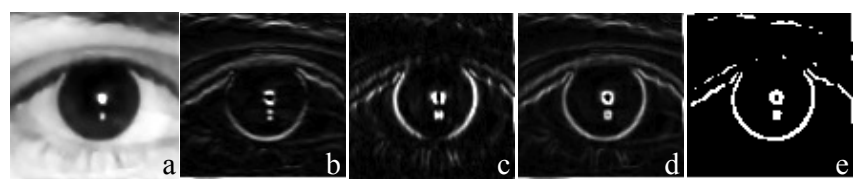

Figure 3. SWT-based edge detection. In (a), the input image, (b) depicts the horizontal detail, (c) depicts the vertical detail, (d) is the combination of horizontal and vertical detail, and (e) is the thresholded version of (d).

of the original input image. Then, it follows to perform a histogram equalization. This is exemplified in the first three rows of Figure 2, i.e., the upper half portion.

Using the filtered and equalized image we approximate the derivative of the image using "Sobel" for the red channel image and "Prewitt" for the green and blue channel images respectively. Then the image is binarized to analyze the edges. The selection of the best derivative approximation methods was determined experimentally.

\section{B. Wavelet-based filtering}

Similarly, this research filters the input RGB image with purposes of denoising using a 2D discrete stationary wavelet transform (SWT) at two decomposition levels, $L=2$. Essentially, SWTs are non-decimated discrete wavelet transforms (DWTs) which preserve the original size of the image [4]. In this implementation of SWTs we use "haar" wavelets as they demonstrated to be better in preliminary experiments. An SWT can be calculated faster if we resize the image such that its size is a factor of $2^{L}$. Thus, we can calculate the new size as follows:

$$
\{\widehat{M}, \widehat{N}\}=\left\{\left\lceil\frac{M}{2^{L}}\right\rceil \times 2^{L},\left\lceil\frac{N}{2^{L}}\right\rceil \times 2^{L}\right\},
$$

where $\widehat{M} \geq M$ and $\widehat{N} \geq N$ denote the new size of an $\widehat{M} \times \widehat{N}$ image. The image is resized using bicubic interpolation. The parameters required for the thresholding of the SWT coefficients are estimated with the traditional method of Donoho [7]. The denoised image is then equalized as described in Section II.

Using the denoised and equalized image, we exploit the SWT one more time in order to approximate the first derivative of the image for edge-detection purposes. In this case the SWT is applied at one single decomposition level, $L=1$, using a "haar" wavelet. The "horizontal" and "vertical" details are combined to produce the derivative approximation. Figure 3 exemplifies the process of having a denoised equalized image (a), and its SWT horizontal detail (b) and its vertical detail (c); the combination of both horizontal and vertical, (d), is an approximation to the first derivative. Finally the combined detail is thresholded to produce a binary image with edges, (e). After this, the edge image is resized back to its original $M \times N$ size. 


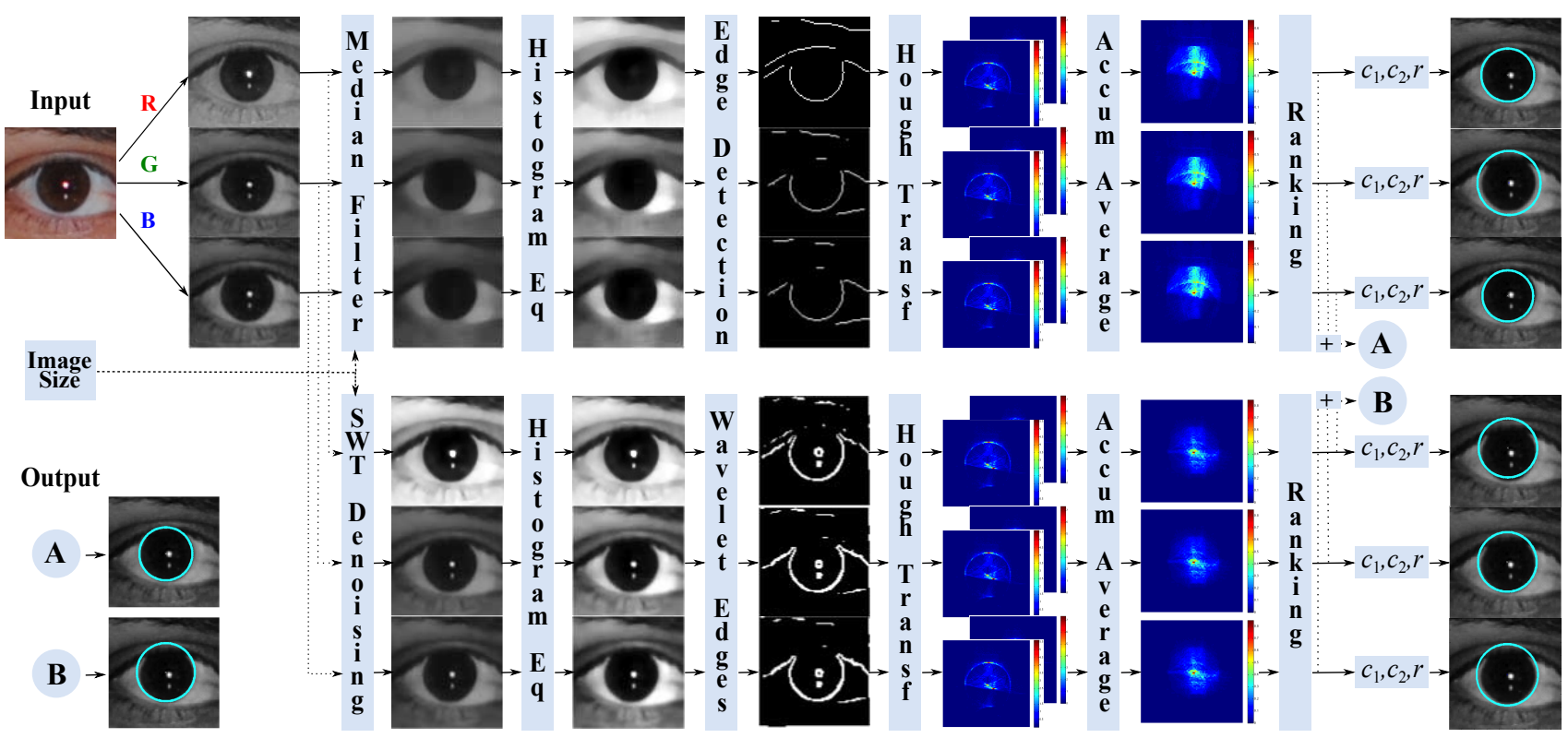

Figure 2. Two methodologies for detecting the iris as region of interest. The upper half implements a median filter whose size depends on the input image. The lower half convolves stationary wavelets to denoise the input images. After denoising, both are processed to equalize their histogram for better edge detection followed by the Hough transform whose accumulator matrix is used to select a center candidate.

\section{Hough transform}

After edge-detection, we use the Hough transform for circles to recognize the likelihood of an image to contain a circle centered in $c_{1}, c_{2}$ with radius $r$, where $c_{1} \in$ $\{0,1, \ldots, M-1\}$ and $c_{2} \in\{0,1, \ldots, N-1\}$. The Hough transform for circles requires an accumulator matrix for each $r$ that is attempted. The accumulator matrix, $\mathbf{A}\left(n_{1}, n_{2}\right)$, has the same size of the input image, $M \times N$, where each element in the matrix contains the number of "votes" for the hypothesis that in that position exists a circle of radius $r$. Let us consider the position $\mathbf{A}\left(n_{1}, n_{2}\right)$ and radius $r$; the Hough transform will understand $\left\{n_{1}, n_{2}\right\}$ as the center $\left\{c_{1}, c_{2}\right\}$ with radius $r$ and will add all $a_{1}, a_{2}$ values that satisfy the circle equation $r^{2}=\left(a_{1}-c_{1}\right)^{2}+\left(a_{2}-c_{2}\right)^{2}$, and will set that value in the position $\mathbf{A}\left(n_{1}, n_{2}\right)$ the process is repeated for every single element in $\mathbf{A}$. At the end, the accumulator matrix $\mathbf{A}$ will represent the likelihood of having a circle of radius $r$ at any position in the image.

Since we are interested in the region delimited by the iris, we limited the radius search to the following monotonic sequence:

$$
r \in\left\{1,2, \ldots,\left\lfloor\frac{\max (M, N)}{2}\right\rfloor\right\} .
$$

In this manner, the radius search space is constrained to search for circles of at most the size of the image. There will be a number of $\left\lfloor\frac{\max (M, N)}{2}\right\rfloor$ accumulator matrices. The maximum value of each accumulator represents the best chance that a circle exists at that position for that radius. Nonetheless, if we average all accumulator matrices into one matrix, $\widehat{\mathbf{A}}\left(n_{1}, n_{2}\right)$, this new accumulator matrix will contain a better representation of the likelihood of circles. The process of averaging reduces the effect of unwanted center candidates, e.g., circle candidates that only occur once in a while, and preserves "good" candidates, e.g., concentric circles and circles that are frequently receiving high number of votes at different (and usually subsequent) radii. This process is exemplified in Figure 2.

The matrix $\widehat{\mathbf{A}}$, if properly normalized, can be understood as a 2D probability density function (PDF). Such PDF can help us rank the positions according to their likelihood to contain a circle. Thus, we followed to use this approximate PDF to rank all the accumulator matrices, $\mathbf{A}$, for the different radii. From all the ranked accumulator matrices we select the position with the highest value, storing its position $c_{1}, c_{2}$ and its corresponding radius $r$. Every single channel will provide its own center candidate, which allows us to propose a fourth candidate based on the average position and radius of all three (RGB) center candidates. This is also exemplified in Figure 2 with connectors (A) and (B). In fact we observed that, in some cases, these candidates, produced by averaging the other three candidates, might be better solutions than individual channel solutions; this is discussed in the next section.

\section{EXPERIMENTS AND RESULTS}

We performed experiments using the datasets described in Section II. The experiments consist of taking an input image and process it to obtain its center candidates, $\left\{\hat{c}_{1}, \hat{c}_{2}, \hat{r}\right\}$, as exemplified in Figure 2. Every input image will produce 
Table I

CROSSVALIDATION ERROR RATE ANALYSIS WITH RESPECT TO THE ESTIMATED CENTER AND RADIUS

\begin{tabular}{ccccccccc}
\hline \multirow{2}{*}{ Error } & \multicolumn{4}{c}{ Median-based } & \multicolumn{4}{c}{ Wavelet-based } \\
& $\mathrm{R}$ & $\mathrm{G}$ & $\mathrm{B}$ & $\mathrm{C}$ Comb. & $\mathrm{R}$ & $\mathrm{G}$ & $\mathrm{B}$ & Comb. \\
\hline Average $\epsilon_{c}$ & $\mathbf{0 . 0 4 7 2}$ & 0.0567 & 0.0630 & 0.0476 & 0.0522 & 0.0554 & 0.0606 & $\mathbf{0 . 0 5 0 7}$ \\
Average $\epsilon_{r}$ & 0.2441 & 0.2387 & 0.2532 & $\mathbf{0 . 2 1 4 9}$ & 0.3747 & 0.3916 & 0.3740 & $\mathbf{0 . 3 2 4 3}$ \\
\hline
\end{tabular}

eight outputs: the first four correspond the median-based approach, i.e., $\mathrm{R}, \mathrm{G}, \mathrm{B}$, and the combined result; and the last four correspond to the wavelet-based approach, respectively. Each output, is compared to the target value in two different ways; one is based on the center and the other based on the radius; both are shown in Table I and explained next.

The first comparison is based on the distance from the target (its true center) to its calculated center. The measure for comparison is the weighted Euclidean distance given by

$$
\epsilon_{c}=\frac{\sqrt{\left(c_{1}-\hat{c}_{1}\right)^{2}+\left(c_{2}-\hat{c}_{2}\right)^{2}}}{\sqrt{(M-r)^{2}+(N-r)^{2}}}
$$

where the denominator accounts for the worst possible scenario, i.e., the largest possible error. In this manner we can make a fair comparison of all errors independently of the input size. The range of $\epsilon_{c}$ is in $[0,1]$. The first row in Table I shows the cross-validation error for $\epsilon_{c}$; the table also shows in bold font the lowest errors for both approaches. Notice how the red channel, in average, produces the lowest error rate with 0.0472 (which is a $4.72 \%$ error); however, note that the error of the three channels combined for the median-filtering approach is also very low 0.0476. The Friedman's test was applied rejecting the null-hypothesis (that all methodologies perform the same), and confirming that the study is statistically significant with $p<0.1 \times 10^{-5}$.

A second comparison measures the level of overlap between the true circle of radius $r$ and the estimated circle of radius $\hat{r}$. The area of the intersection, $T$, between two circles can be easily estimated under the following considerations: a) If the target circle and the estimated circle do not overlap, then $T=0 ; b)$ if both circles fully overlap, then the overlap area is computed as $T=\pi \min (r, \hat{r})^{2}$; and c) if both circles partially overlap, then the intersection area is computed and assigned to $T$. Thus, the radius error based on the intersection intersection can be estimated as follows:

$$
\epsilon_{r}=1-\frac{T}{\pi r^{2}}
$$

where the denominator describes the maximum possible intersection area, which is the area of the true circle. The range of $\epsilon_{r}$ is between $[0,1]$. The second row of Table I presents the crossvalidation error for $\epsilon_{r}$, based on the above criteria. Clearly, the combined approach using median filtering exhibits the lowest average error and it is the best ranked methodology overall. The Friedman's test shows that the study is statistically significant with $p<0.00001 \times 10^{-5}$, rejecting the null hypothesis.

\section{CONClusion}

This research introduces two approaches for finding the smallest circle containing the iris: a median filter-based approach and a wavelet approach in conjunction with the Hough transform. From the experiments we observed that the median filter approach produces similar results than the wavelet-based approach in finding the coordinates of the center of the true circle; however, the median-filter approach seems more robust in finding a better radius. Nonetheless, both methodologies present novel alternatives towards an automatic system for detecting leukocoria in infants with retinoblastoma. This research also presents an alternative image analysis approach in the denoised wavelet domain aiming to find a circular shape. Further studies will analyze the Hough transform for elipses.

\section{ACKNOWLEDGMENT}

This work was supported in part by the National Council for Science and Technology (CONACyT), Mexico, under grant 193324/303732. Thanks to Dr. Erich Baker for his support in providing and gathering data.

\section{REFERENCES}

[1] J. C. Canzano and J. T. Handa, "Utility of pupillary dilation for detecting leukocoria in patients with retinoblastoma," $\mathrm{Pe}$ diatrics, vol. 104, no. 4, pp. e44-e44, 1999.

[2] H. Demirci, C. L. Shields, J. A. Shields, S. G. Honavar, and R. C. Eagle, "Leucocoria as the presenting sign of a ciliary body melanoma in a child," British Journal of Ophthalmology, vol. 85 , no. 1, pp. 110-110, 2001.

[3] B. G. Haik, L. Saint Louis, M. E. Smith, R. M. Ellsworth, D. H. Abramson, P. Cahill, M. Deck, D. J. Coleman et al., "Magnetic resonance imaging in the evaluation of leukocoria." Ophthalmology, vol. 92, no. 8, pp. 1143-1152, 1985.

[4] X. H. Wang, R. Istepanian, and Y. H. Song, "Microarray image enhancement by denoising using stationary wavelet t." Nano Bioscience, IEEE Trans. on, vol. 2, no. 4, pp. 184-189, 2003.

[5] J. Illingworth and J. Kittler, "The adaptive hough transform," Pattern Analysis and Machine Intelligence, IEEE Transactions on, vol. PAMI-9, no. 5, pp. 690-698, 1987.

[6] H. Yuen, J. Princen, J. Illingworth, and J. Kittler, "Comparative study of hough transform methods for circle finding," Image and Vision Computing, vol. 8, no. 1, pp. 71 - 77, 1990.

[7] D. Donoho, "De-noising by soft-thresholding," Information Theory, IEEE Trans. on, vol. 41, no. 3, pp. 613-627, 1995. 